\title{
Evaluation of Pattern of Abdominal Surgical Emergencies
}

Dr. Md. Anisur Rahman ${ }^{1}$, Dr. ASM Rezbanul Haque ${ }^{2}$, Dr. Bablu Kumar Saha ${ }^{3}$, Dr. Upendra Nath Ray ${ }^{4}$, Dr. Md Mahfuzul Haque ${ }^{5 *}$, Dr. Md Abdus Sattar ${ }^{5}$, Dr. Most Arifa Begum ${ }^{6}$, Dr. Miratul Jesmin ${ }^{6}$, Dr. Md Golam Shorwer ${ }^{7}$, Dr. Shamima Najma ${ }^{8}$

${ }^{1}$ Resident Surgeon, Paediatric Surgery, Rangpur Medical College Hospital, Bangladesh

${ }^{2}$ Registrar, Paediatric Surgery, Rangpur Medical College Hospital, Bangladesh

${ }^{3}$ Associate Professor, Paediatric Surgery, Rangpur Medical College, Bangladesh

${ }^{4}$ Assistant Registrar, Paediatric Surgery, Rangpur Medical College, Bangladesh

${ }_{5}^{5}$ Assistant Professor, Paediatric Surgery, Rangpur Medical College Hospital, Bangladesh

${ }^{6}$ Medical Officer, Rangpur Medical College Hospital, Bangladesh

${ }^{7}$ Senior consultant Anaesthesiology, M Abdur Rahim Medical College \& Hospital, Dinajpur, Bangladesh

${ }^{8}$ Consultant, Prime Medical College, Rangpur, Bangladesh

DOI: $10.36347 /$ sasjs.2020.v06i10.005

| Received: 22.09.2020 | Accepted: 01.10.2020 | Published: 21.10.2020

*Corresponding author: Dr. Md Mahfuzul Haque

Abstract

Original Research Article

Objective: In this study our main goal is to evaluate the pattern of abdominal surgical emergencies. Method: This cross-sectional descriptive study was carried out Department of Surgery, Rangpur Medical College Hospital, Rangpur from 12months (January 2018 to December 2018). Total of 250 admitted patients of acute abdominal conditions requiring emergency surgery in department of Surgery, Rangpur Medical College Hospital, Rangpur were included in this study. Results: During the study, according to verification of clinical diagnosis $92.74 \%$ cases the clinical diagnosis coincides with per-operative(confirmatory) diagnosis. $92.74 \%$ cases the clinical diagnosis coincides with peroperative(confirmatory) diagnosis.Among the patients with plain X-ray of abdomen, multiple air fluid level present in 60 cases, among the patients with ultrasonogram of whole abdomen, acute appendicitis found in 18 cases and among the patients, complete blood count shows neutrophilic leukocytosis is about 118 cases. Conclusion: From our study we can conclude that, non-traumatological abdominal surgery emergencies represent a non-neglected proportion of admission in surgery, patients concerned are mostly young adults with predominance of male or female.The most incriminated pathology is the acute appendicitis. Adhesion, colonic growth and sigmoid volvulus are the frequent etiology of Intestinal obstruction.

Keywords: Acute abdomen, bowel obstruction, emergency surgery.

Copyright (C) 2020 The Author(s): This is an open-access article distributed under the terms of the Creative Commons Attribution 4.0 International License (CC BY-NC 4.0) which permits unrestricted use, distribution, and reproduction in any medium for non-commercial use provided the original author and source are credited.

\section{INTRODUCTION}

In low- and middle-income countries, at least $60 \%$ of the surgical operation performed are for emergency, contrary of widespread report has been shown that the provision of treatment which is often life saving for those patients can be the advice [1].

Emergency surgery can be defined as unscheduled surgery [2]. Surgical emergencies represent more than $50 \%$ of surgical admissions and constitute a major part of the surgeons' workload in most parts of the world. Proportion of non-trauma surgical emergencies is reported to be between $30 \%$ and $57 \%$ with more than half requiring surgical intervention. This is higher than $30 \%-50 \%$ of trauma patients who will require emergency surgery [3]. Nontraumatological abdominal surgical emergencies represented $19.8 \%$ of admission in surgery department. Principle etiology of non-traumatological abdominal surgical emergencies were appendicitis (47.8\%), peritonitis $(21.2 \%)$ and intestinal obstruction (19.2\%) [4]. Fifty six patient had a clinical diagnosis of appendicitis and out of them 44 patients underwent appendicectomy within the first 24 hours [5].

Bowel obstruction is one of the most common causes of abdominal pain in the elderly (12-25\%) and is second only to biliary disease is an indication for emergency abdominal surgery. The etiology of small bowel obstruction is predominantly surgical adhesions $(50-70 \%)$ followed by incarcerated hernia $(15 \%)$ [6]. In contrast, in patients younger than 60 years acute appendicitis is the most common surgically correctable cause, accounting for approximately $25 \%$ of cases [7]. 



Fig-1: The location and character of pain are helpful in the differential diagnosis of the acute abdomen [8]

As acute abdomen is common presentation of surgical emergency, early diagnosis of surgical diseases and emergency surgical intervention is done. Different clinical series available have identified the pattern of abdominal surgical emergencies in elderly and children. But there is no data available in relation to the abdominal surgical emergencies in adults. In this study our main goal is to evaluate the pattern of abdominal surgical emergencies.

\section{OBJECTIVE \\ General objective}

- To assess the pattern of abdominal surgical emergencies.

\section{Specific objective:}

- To identify lab findings of investigations

- To detect per-operative findings of the patients.

\section{METHODOLOGY}

\begin{tabular}{|l|l|}
\hline Type of study & Cross sectional descriptive study. \\
\hline Place of study & Department of Surgery, Rangpur MedicalCollege Hospital, Rangpur \\
\hline Study period & 12 months (January 2018 to December 2018). \\
\hline Study population & $\begin{array}{l}\text { Total of } 250 \text { admitted patients of acute abdominal conditions requiring emergency surgery in } \\
\text { department of Surgery, Rangpur Medical College Hospital, Rangpurwere included in this study. }\end{array}$ \\
\hline $\begin{array}{l}\text { Sampling } \\
\text { technique }\end{array}$ & Purpose sampling. \\
\hline
\end{tabular}

\section{Inclusion Criteria}

- $\quad$ Age between 18-60 years of both sexes.

- Patients with abdominal complaints requiring emergency surgery.

- Chronic abdominal pain with an acute attack.

- Positive findings in USG and X-ray abdomen.

\section{Exclusion Criteria}

- Patient presented with acute abdomen of traumatic origin.

- Elective surgery not linked to an emergency surgery assessment.
- Acute abdomen due to Obstetric and Gynecological causes.

- Invasive abdominal procedure within the previous 30 days.

\section{METHOD}

Detailed information was obtained in each case according to protocol. Complete history was taken either from patient or accompanying attendants. Though physical examination was done, relevant investigations $\&$ operation notes were collected. All the information was recorded in the fixed protocol. 


\section{Statistical Analysis}

Collected data was classified, edited, coded \& entered into the computer using computer software SPSS (Statistical Package for Social Science Versions 24.0).

\section{RESULTS}

In Table-1 shows sociodemographic characteristic of the patients where $50 \%$ of the emergency patients were in the age group 18-30 years and $73.8 \%$ were female. The following table is given below in detail:

Table-1: Sociodemographic characteristic of the patients

\begin{tabular}{|l|l|l|}
\hline Age group & Number $(\mathbf{n})$ & Percentage $(\%)$ \\
\hline 18-30 years & 124 & 50 \\
\hline 31-45 years & 52 & 20.97 \\
\hline 46-60 years & 72 & 29.03 \\
\hline Sex & Number $(\mathbf{n})$ & Percentage $\mathbf{( \% )}$ \\
\hline Male & 183 & 73.8 \\
\hline Female & 65 & 26.2 \\
\hline Occupation & Number & Percentage $(\%)$ \\
\hline Employed & 30 & 12.10 \\
\hline Unemployed & 10 & 4.03 \\
\hline Business & 15 & 6.05 \\
\hline Farmer & 40 & 16.13 \\
\hline Student & 88 & 35.48 \\
\hline Housewife & 39 & 15.73 \\
\hline Day labourer & 26 & 10.48 \\
\hline
\end{tabular}

In Figure-2 shows distribution of the patients according to verification of clinical diagnosis where $92.74 \%$ cases the clinical diagnosis coincides with per- operative (confirmatory) diagnosis. The following figure is given below in detail:

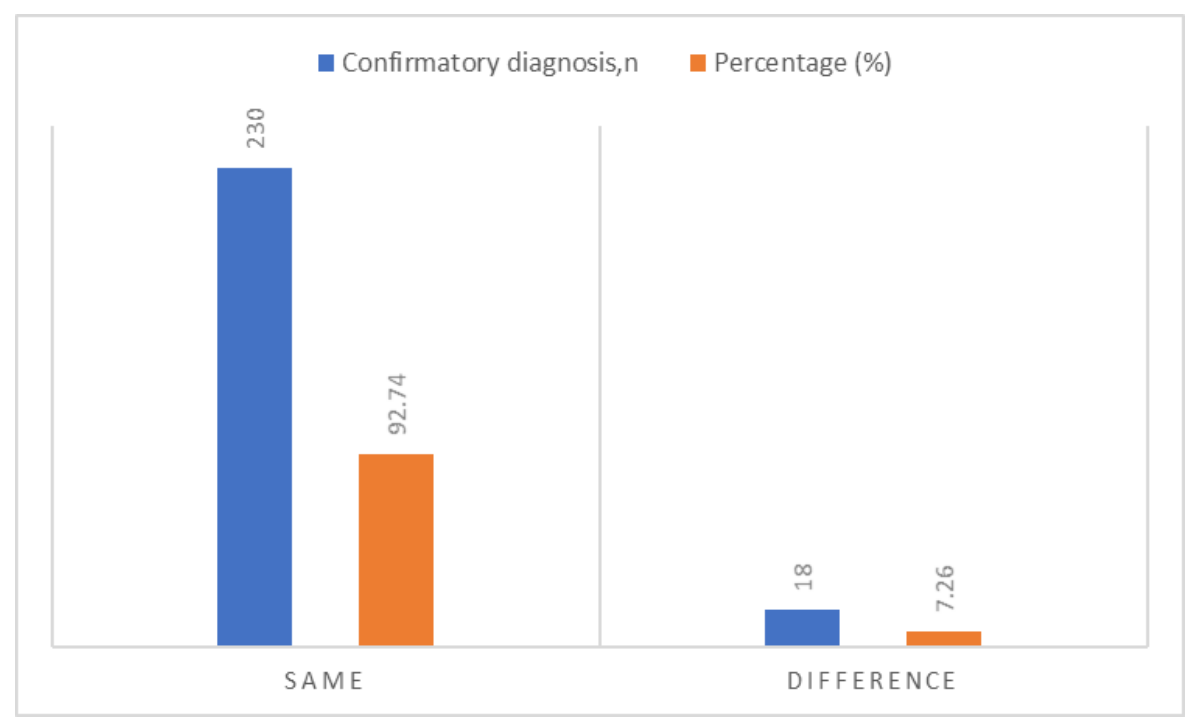

Fig-2: Distribution of the patients according to verification of clinical diagnosis

In table-2 shows pattern of abdominal surgical emergencies where acute appendicitis was $42.34 \%$, intestinal obstruction followed by $24.60 \%$, perforation of GCHV $19.76 \%$ and it was statistically significant (p $<0.05)$. The following table is given below in detail: 
Table-2: Pattern of abdominal surgical emergencies $(\mathbf{n}=\mathbf{2 4 8})$

\begin{tabular}{|c|c|c|c|c|}
\hline Disease & Number & Percentage & $\mathrm{X}^{2}$ value at $95 \% \mathrm{CI}$ & Probability \\
\hline Acute Appendicitis & 105 & 42.34 & \multirow{6}{*}{41.33} & \multirow{6}{*}{$\mathrm{P}<0.05^{*}$} \\
\hline Intestinal Obstruction & 61 & 24.60 & & \\
\hline Perforation of GCHV & 49 & 19.76 & & \\
\hline Appendicular abscess & 07 & 2.82 & & \\
\hline Burst Appendicitis & 14 & 5.64 & & \\
\hline Empyema Gall bladder & 12 & 4.84 & & \\
\hline
\end{tabular}

In $\mathrm{X}^{2}$ test of significance of difference $*=\mathrm{P}<0.05$

In Figure-3 shows distribution of patients on the basis of findings of investigations whereamong the patients with plain X-ray of abdomen, multiple air fluid level present in 60 cases, among the patients with ultrasonogram of whole abdomen, acute appendicitis found in 18 cases and among the patients, complete blood count shows neutrophilic leukocytosis is about 118 cases. The following figure is given below in detail:

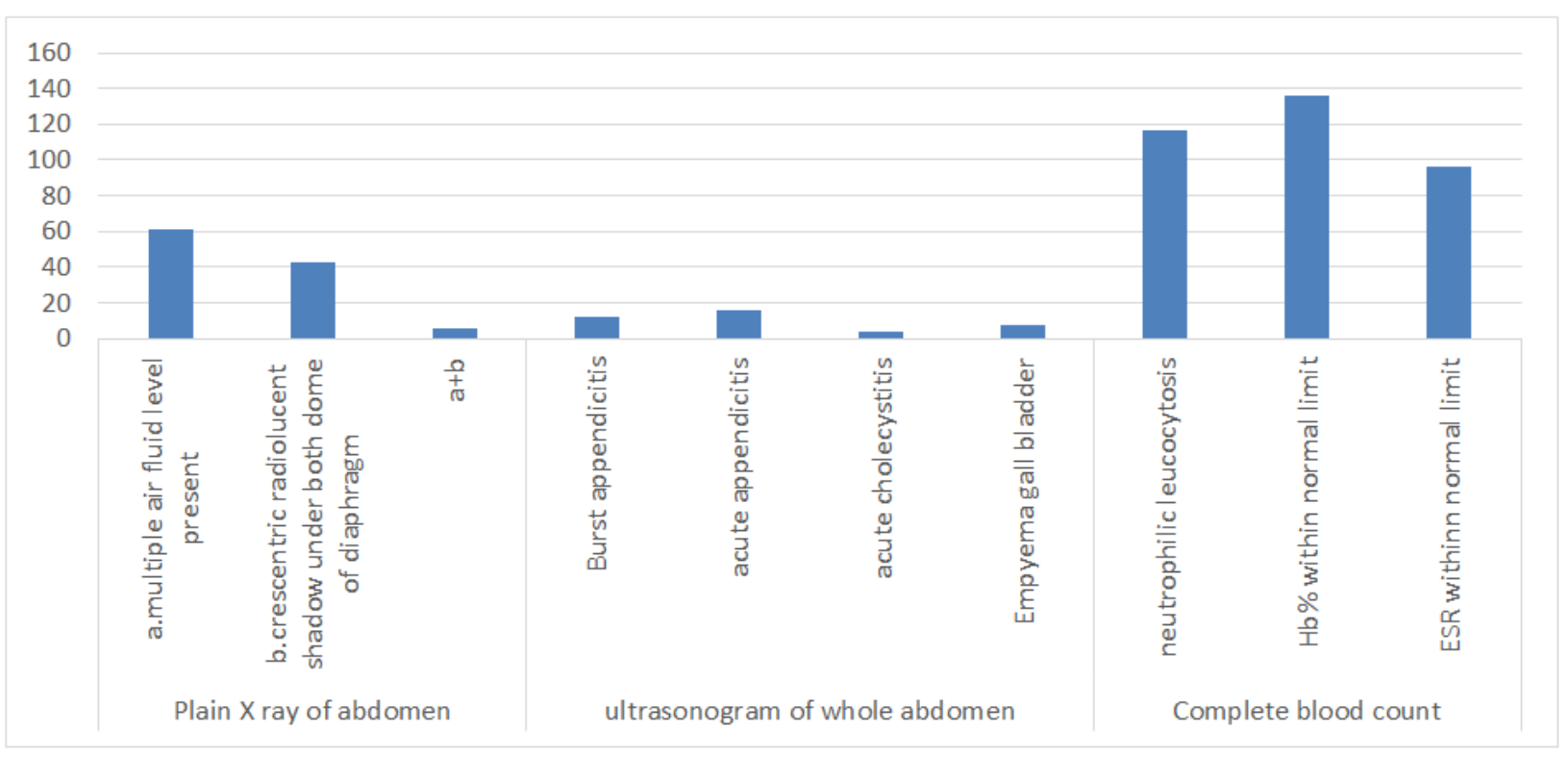

Fig-3: Distribution of patients on the basis of findings of investigations

In Table-3 shows distribution of patients on the basis of time interval between admission and Operations where $57.26 \%$ cases operation was done within 24 hours of admission. The following table is given below in detail:

Table-3: Distribution of patients on the basis of time interval between admission and Operations (n-248)

\begin{tabular}{|l|l|l|}
\hline Time interval between admission and operation & Number $(\mathbf{n})$ & Percentage $(\%)$ \\
\hline 6 hours & 08 & 3.23 \\
\hline 12 hours & 38 & 15.32 \\
\hline 24 hours & 142 & 57.26 \\
\hline 48 hours & 37 & 14.92 \\
\hline 72 hours & 23 & 9.27 \\
\hline
\end{tabular}

In Figure-4 shows distribution of patients on the basis of per-operative findings. Among the patient with appendicular lesion, obstructive appendicitis is about 55, among the perforated cases anterior wall of duodenal perforation is the commonest (32), among the intestinal obstruction, sigmoid colon is the common site of obstruction (16). The following figure is given below in detail: 


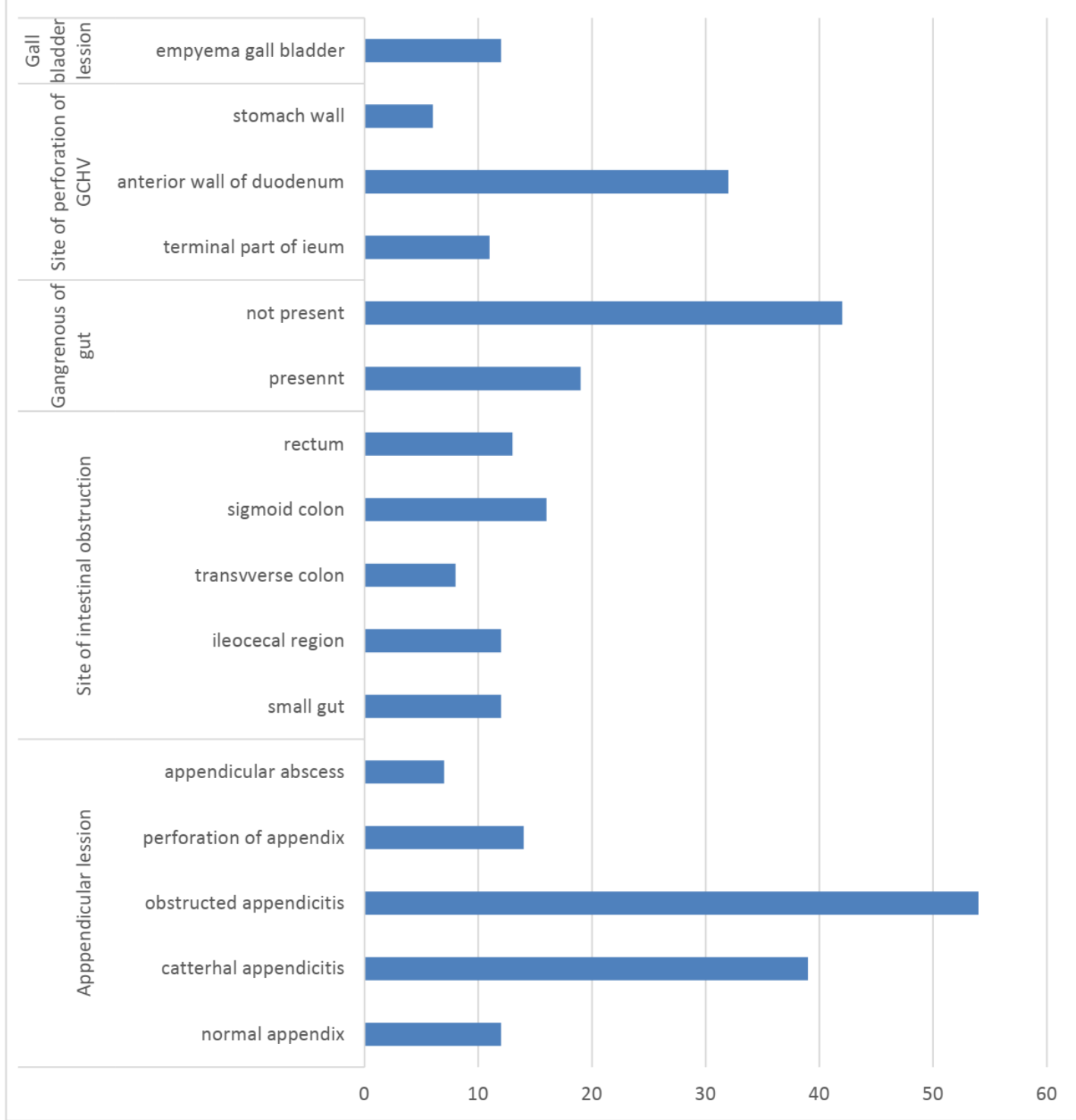

Fig-4: Distribution of patients on the basis of per-operative findings

In Table-4 shows causes of Intestinal obstruction (n -61) colonic growth $(37.70 \%)$, volvulus $(27.87 \%)$ and bands and adhesion $(21.31 \%)$ were the most common causes of intestinal obstruction. The following table is given below in detail:

Table-4: Causes of Intestinal obstruction (n -61)

\begin{tabular}{|l|l|l|}
\hline Intestinal Obstruction & Number & Percentage (\%) \\
\hline Colonic growth & 23 & 37.70 \\
\hline Bands and adhesion & 13 & 21.31 \\
\hline Volvulus & 17 & 27.87 \\
\hline Obstructed Inguinal hernia & 6 & 9.84 \\
\hline Intussusception & 2 & 3.28 \\
\hline
\end{tabular}

\section{DISCUSSION}

In this study age distribution was $18-30$ years $124(50 \%), 31-45$ years $52(20.97 \%)$ and 46-60 years 72 $(29.03 \%)$. Here age was statistically significant $(\mathrm{p}<0.05)$ with abdominal surgical emergencies. Here mean age was 35.35. A similar study was conducted by one study showed highest incidence was found in patients between 21-30 years i.e.27.81\% [6]. Another study Ajay Malviya et al., showed highest incidence $(27.64 \%)$ of acute non traumatic emergency was seen in 21-30 years age group [9]. Similar trend of age was noted other study [10].

In this current study male were $183(73.8 \%)$ and female $65(26.2 \%)$ and male female ratio was almost 2.82:1. A similar study was conducted where he 
found male were $70(71.43 \%)$ and $28(28.57 \%)$ females with a male: female ratio of 2.5:1. 11 [11]. Another report conduct a similar study where he found a total of 586 patients presented with non-traumatic acute abdomen of which there were $412(70.30 \%)$ males and $174(29.63 \%)$ females with Asian General Hospital male to female ratio of 2.3:1 [10].

In this study occupation distributed among the patient were student $35.48 \%$ (n-88), farmer $16.13 \%$ (n40), housewife $15.73 \%$ (n-39), employed $12.10 \%$ (n$30)$, day laborer $10.48 \%$ (n-26), business $6.05 \%$ (n-15) and unemployed $4.03 \%$ (n-10).

In this study the socioeconomic status of respondents were lower class $62.10 \%$ (n-154) middle class $37.90 \%$ (n-94). The socioeconomic status of respondents was statistically non-significant with abdominal surgical emergencies.

Acute appendicitis was found to be the most common abdominal surgical emergency in our study which was $105(42.34 \%)$ followed by Intestinal obstruction 61(24.60\%), Perforation of Gas Containing Hollow Viscus (GCHV) 49(19.76\%), Appendicular abscess 07(2.82\%),Burst Appendicitis 14(5.64\%), Empyema Gall bladder 12(4.84\%).The result is statistically significant $(\mathrm{p}<0.05)$. However appendicitis was found to be the leading cause in several studies [3, 12]. A similar study was conducted by one study Abdurrahman $\mathrm{Z}$ et all where he found that the most common surgical emergencies are acute appendicitis $52 \%$ (n-122), followed by Intestinal obstruction $26 \%$ (n-62), Perforation of Peptic Ulcer [13].

Disease 09\% (n-21) and others 13\% (n-31). Another study was found emergency surgery for acute abdomen constituted two-thirds of non-trauma operations with acute appendicitis/ruptured appendix $(28 \%) \&$ Intestinal obstruction $(28 \%)$ being the most diagnosis [3]. Another study was conducted where he found that Appendicitis is a common surgical emergency \& in this series represented about $9.4 \%$ (n56) performed which is the highest in general surgical emergencies [5]. Another study found the most frequent etiology is the acute appendicitis $47.8 \%$ followed by acute peritonitis, acute intestinal ischemia with respectively $21.2 \%$ and $10.2 \%$ [4].

Acute appendicitis was the most frequent cause $(55.20 \%)$ followed by acute cholecystitis $(29.40 \%)$. Study done by Another study showed the most common causes of acute abdomen was acute appendicitis accounting for $35 \%$ admission and intestinal obstruction was second leading cause accounting for $28.5 \%$ cases [10].

Acute appendicitis was the most common causes of acute abdomen in this study consistent with other studies carried in England and locally.Compared to other studies we had higher incidence of acute appendicitis $(46 \%)$, the higher incidence could be attributed due to the fact that in our studies medical. Urological and gynecological cases are excluded.

In this study, intestinal obstruction was the next most common abdominal surgical emergencies (24.60\%).The leading causes of intestinal obstruction in this series were colonic growth (23), sigmoid volvulus (17), and band and adhesion (13). A similar study was found small bowel obstruction due to adhesion and sigmoid volvulus were the leading cause of Intestinal obstruction each accounting for 27\% (n-17) [13]. Another study found complicated abdominal wall hernia was the cause of obstruction in $52 \%$, peritonitis followed perforated peptic ulcer in $7.2 \%$ [3].

This study found, appendicular lesion were the most observed lesion $(50.81 \%)$ during per operative findings followed by acute intestinal obstruction $(24.60 \%)$. A similar study was found that according to per operative lesion observed appendicular lesion were the most observed lesion $(54.7 \%)$ followed by acute intestinal occlusion, perforation of digestive tract with per operatively $19.2 \% \& 8.8 \%$ [4].

In this study showed that, surgery performed within 24 hours of admission was 75.81bn u87\%. Similar study was done and they found if surgery performed within 24 hours of disease onset, mortality should be much lower than 10 percent [1].

\section{CONCLUSION}

From our study we can conclude that, nontraumatological abdominal surgery emergencies represent a non-neglected proportion of admission in surgery, patients concerned are mostly young adults with predominance of male or female. The most incriminated pathology is the acute appendicitis. Adhesion, colonic growth and sigmoid volvulus are the frequent etiology of Intestinal obstruction.

\section{REFERENCE}

1. McCord C, Ozgediz D, Beard JH, Debas HT. General surgical emergencies. 2014; 4:61-74.

2. Clinical Excellence division, Healthcare Improvement Unit, Emergency Surgery Access Guideline, QH-GDL, 2017; 2:440.

3. Ibrahim NA, Oludara MA, Ajani A, Mustafa I, Balogun R, Idowu O, Osuoji R, Omodele FO, Aderounmu AO, Solagberu BA. Non-trauma surgical emergencies in adults: Spectrum, challenges and outcome of care. Annals of Medicine and Surgery. 2015 Dec 1;4(4):325-30.

4. Valimungighe MM, Bunduki GK, Kuyigwa MN, Ahuka OL. Aetiologies of non-traumatological abdominal surgery emergencies in butembo, democratic republic of congo. International Journal of Current Advanced Research, 2015;4(9):357-359. 
5. Scurr JH. Surgical emergencies in general practice. The Journal of the Royal College of General Practitioners. 1979 Dec 1;29(209):744-7.

6. Hendrickson M, Naparst TR. Abdominal surgical emergencies in the elderly. Emergency medicine clinics of North America. 2003 Nov 1;21(4):93769.

7. Karnath B, Mileski W. Acute abdominal pain. Hospital Physician. 2002 Nov;38:45-50.

8. Hardy A, Butler B, Crandall M. The evaluation of the acute abdomen. In Common problems in acute care surgery (pp. 19-31). Springer, New York, NY. 2013.

9. Malviya A, Hussain A, Bulchandani HP, Bhardwaj G, Kataria S. A comprehensive study on acute non-traumatic abdominal emergencies. International Surgery Journal. 2017 Jun 22;4(7):2297-302.
10. Memon AA, Bhutto AA, Shaikh GS, Jokhio A, Soomro QA. Spectrum of diseases in patients with non-traumatic acute abdomen. J Liaquat Uni Med Health Sci. 2008 Sep;7(3):180-3.

11. Jain R, Gupta V. A prospective study of epidemiology and clinical presentation of nontraumatic acute abdomen cases in a tertiary care hospital of central India. International Surgery Journal. 2016 Dec 13;4(1):242-5.

12. Kotiso B, Abdurrahman Z. Pattern of acute abdomen adult patients in Taikur Abessa Teaching Hospitital, Addis Ababa Ethiopia. East and Central African Journal of Surgery, 2017; 4(12):47-52.

13. Abdul Mohsen A. Al Mulhim, Emergency general surgical admissions. Prospective institutional experience in non-traumatic acute abdomen: implications for education, training and service. Saudi Med. J. 2006;27(11):1674-9. 